DOI:10.18027 / 2224-5057-2017-7-3-62-69

\title{
Эффективность ниволумаба в лечении метастатической меланомы
}

\author{
Г.Ю. Харкевич, Л.В. Демидов
}

НИИ клинической онкологии ФГБУ «НМИЦ онкологии им. Н. Н. Блохина», Москва, Россия

\begin{abstract}
Резюме: Появление нового класса иммуно-онкологических препаратов - ингибиторов блокаторов иммунного ответа - позволило достигнуть прогресса в терапии метастатической меланомы. Первым препаратом, продемонстрировавшим улучшение общей выживаемости у пациентов, ранее получавших лечение, был ипилимумаб. Ниволумаб - представитель нового класса препаратов, ингибитор рецептора PD-1 Т-лимфоцитов. Клиническая эффрективность ниволумаба в дозе 3 мг/кг каждые 2 недели изучалась в трех исследованиях 3 фразы. На фроне терапии ниволумабом в монорежиме медиана общей выживаемости (ОВ) составила 37,6 мес., а в комбинации с ипилимумабом - не достигнута при минимальном периоде наблюдения 36 мес. Трехлетняя ОВ была 58\% в группе комбинации и $52 \%$ в группе монотерапии ипилимумабом в сравнении с $34 \%$ в группе ипилимумаба. Частота объективных ответов составила 45\% и 58\% соответственно в монорежиме и в комбинации с ипилимумабом. В отдельных подгруппах пациентов (экспрессия PD-L1 <1\%, BRAF мутированные пациенты) комбинация демонстрирует лучшие результаты. Профиль безопасности ниволумаба удовлетворительный, частота нежелательных реакций (HP) 3-4 степени по данным совокупного анализа составляет 10\%. В группе комбинации частота НP 3-4 степени была $55 \%$, однако большинство НР отвечают на терапию стероидами и купируются при следовании разработанным алгоритмам. Таким образом, ингибиторы PD-1, и в частности ниволумаб, как в режиме монотерапии, так и в комбинации с ипилимумабом, становятся новым стандартом терапии метастатической меланомы в первой линии, независимо от статуса мутации BRAF или уровня экспрессии PD-L1.
\end{abstract}

Ключевые слова: метастатическая меланома, ингибиторы блокаторов иммунного ответа, иммунотерапия, PD-1, CTLA-4

\section{Введение}

Меланома является злокачественной опухолью, которая развивается из меланоцитов. За последние десятилетия заболеваемость меланомой в развитых странах неуклонно растет $[1,2,3]$, и по темпам прироста данного показателя меланома занимает одно из лидирующих мест (прирост заболеваемости ежегодно составляет 3-7\%) [4, 5]. В России с 2012 по 2016 г. заболеваемость меланомой увеличилась на 31 \% (с 8182 случаев в 2012 г. до 10347 в 2016 г.) [6].

До недавнего времени прогноз пациентов с метастатической меланомой оставался крайне неблагоприятным. Менее $5 \%$ пациентов с диссеминированной меланомой переживали 5 -летний период. Хирургическое лечение может быть эффективной опцией для ограниченного числа пациентов с солитарными метастазами, во всех остальных случаях операция, как и лучевая терапия, носит паллиативный характер. Химиотерапия с использованием дакарбазина и других цитостатических агентов ни в одном из исследований не продемонстрировала улучшение общей выживаемости больных [7, 8, 9].

В последние годы в практику лечения метастатической меланомы вошли новые препараты, такие как BRAF [13] и MEK [14] ингибиторы, которые воздействуют на МАРК-киназный сигнальный путь. В рандомизированных исследованиях эти препараты показали свое преиму- щество над химиотерапией, однако их применение ограничено наличием мутации гена BRAF в опухоли $[10,11,12]$.

В настоящее время широко развивается направление, изучающее влияние иммунной системы на канцерогенез и механизмы ускользания опухоли от иммунного надзора $[13,14,15,16]$. Понимание этих механизмов позволило разработать и успешно внедрить в клиническую практику новые иммуноонкологические препараты, получившие название ингибиторов блокаторов иммунного ответа. Блокируя ингибирующие рецепторы на поверхности Т-лимфоцитов, эти моноклональные антитела позволяют активировать ранее подавляемые опухолью пути активации собственного противоопухолевого иммунитета. Ингибиторы блокаторов иммунного ответа уже зарегистрированы во многих странах для лечения метастатических форм различных опухолей, в том числе меланомы, немелкоклеточного рака легкого, почечноклеточного рака, уротелиального рака, плоскоклеточного рака органов головы и шеи, гепатоцеллюлярной карциномы и других. В нашей стране на сегодняшний день зарегистрированы и разрешены к медицинскому применению следующие препараты данной группы: ипилимумаб, ингибитор CTLA-4 рецепторов Т-лимфоцитов, впервые в мире продемонстрировавший увеличение общей выживаемости у пациентов с метастатической меланомой [17], и ингибиторы рецептора программируемой гибели клеток PD-1 - пембролизумаб и ниволумаб [16, 18]. Кроме того, активно изучаются комбинации этих препаратов $[19,20]$ и новые молекулы 
(анти-LAG-3, IDO-ингибиторы, анти-GITR) в комбинации c PD-1 ингибиторами, которые продемонстрировали многообещающие результаты в ранних фазах исследований $[21,22,23]$. Для лечения метастатической меланомы одобрены 2 ингибитора PD-1 - пембролизумаб и ниволумаб.

В данном обзоре будут представлены исследования по эффективности и безопасности применения ниволумаба в лечении метастатической меланомы у взрослых пациентов.

\section{Клиническая эффективность ниволумаба}

\section{Монотерапия ниволумабом ранее получавших лечение пациентов с метастатической меланомой}

Первые данные по безопасности и эффективности монотерапии ниволумабом были получены в исследовании CheckMate 003, фаза 1, в котором ниволумаб применялся для лечения солидных опухолей, в том числе у пациентов с метастатической меланомой [24]. Следует отметить, что все включенные в это исследование больные ранее уже получали лечение, причем $47 \%$ пациентов получили более 3-х линий предшествующей терапии. Частота объективных ответов (ЧОО) оценивалась по модифицированным критериям RECIST 1.0.

$\mathrm{V}$ пациентов с метастатической меланомой, которые получали ниволумаб в различных дозах $(0,1-10$ мг/кг каждые 2 недели), ЧОО составила 28\% (26 из 94 пациентов), при этом ЧОО ниволумаба в дозе 3 мг/кг каждые 2 недели составила 41\% (7 из 17 пациентов). Один пациент (6\%), получавший ниволумаб в дозе 3 мг/кг, и 6 пациентов (6\%), получавших другие дозы ниволумаба, имели длительную стабилизацию заболевания (более 24 недель на момент анализа).

Выживаемость без прогрессирования (ВБП) на 24 неделе составила $55 \%$ и $41 \%$ соответственно на дозе 3 мг/кг и в остальных режимах. Одно- и двухгодичная общая выживаемость (OB) во всей когорте пациентов составила $62 \%$ и $43 \%$ соответственно, а медиана ОВ - 16,8 мес. [24].

В настоящее время доступны данные 5 -летней ОВ пациентов, получавших ниволумаб в дозе 3 мг/кг каждые 2 недели после более чем 3-х линий предшествующей терапии, которая составила $35 \%$ [25].

В регистрационном исследовании 3 фазы CheckMate 037 ниволумаб изучался у пациентов с метастатической меланомой, у которых прогрессия развилась после или на фоне терапии ипилимумабом [26]. 22\% пациентов имели мутацию BRAF и кроме ипилимумаба получали ингибиторы BRAF в предшествующих линиях терапии. Ниволумаб применялся в стандартной дозе 3 мг/кг каждые 2 недели. Группа сравнения получала химиотерапию по выбору

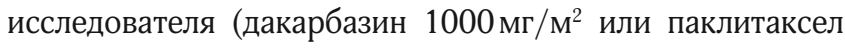

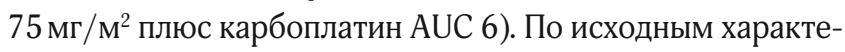

ристикам пациенты были схожими в обеих группах, однако пациентов, имеющих метастазы в головном мозге, было больше в группе ниволумаба (19\% и 14\%), также в группе ниволумаба большее количество пациентов имело уровень ЛДГ выше верхней границы нормы (ВГН) - 51\% и 35\% соответственно. Свыше 73\% пациентов в обеих группах имели 3 и более линий предшествующей терапии.

Основными показателями эффективности в данном исследовании были ЧОО и ОВ. Первичный анализ ЧОО проведен после того, как у 120 пациентов, получавших ниволумаб, период наблюдения составил более 24 недель. Ниволумаб продемонстрировал преимущество над химиотерапией по частоте объективных ответов (32\% против $11 \%)$, включая полные ответы (ПО) у $4 \%$ пациентов в группе ниволумаба. По длительности достигнутых ответов ниволумаб также продемонстрировал преимущество: медиана длительности ответов в группе ниволумаба не была достигнута, а в группе ХТ составила 3,5 месяца. Ниволумаб демонстрирует преимущество в эффективности по частоте объективных ответов, независимо от статуса мутации BRAF и уровня экспрессии PD-L1 [26].

Недавно были опубликованы данные по общей выживаемости пациентов в этом исследовании. При анализе всех рандомизированных пациентов медиана ОВ составила 15,7 мес. в группе ниволумаба и 14,4 мес. в группе ХТ. Одно- и двухлетние показатели ОВ составили соответственно 59\% и 38\% в группе ниволумаба и 55\% и $34 \%$ в группе XT [27].

Отсутствие различий в общей выживаемости между двумя группами, вероятно, связано с тем, что после прогрессирования на химиотерапии $41 \%$ пациентов получил ингибитор PD1 пембролизумаб. Это, несомненно, отразилось на показателях ОВ. При дополнительном анализе с цензурированием по дате начала анти-PD-1 терапии медиана ОВ в группе ниволумаба была выше и составила 16,4 мес., а в группе ХТ - 11,8 мес. (отношение рисков 0,81, 95\% ДИ 0,59-1,1) [27].

Таким образом, у пациентов с метастатической меланомой, ранее получавших лечение, ниволумаб демонстрирует свою эффективность независимо от статуса мутации BRAF и уровня экспрессии PD-L1, а 5-летняя общая выживаемость достигает $35 \%$.

\section{Монотерапия ниволумабом у пациентов с метастатической меланомой, ранее не получавших лечение}

В рандомизированном исследовании 3 фазы CheckMate 066 клиническая эффективность и безопасность ниволумаба изучалась у ранее не получавших терапию пациентов с нерезектабельной или метастатической меланомой без мутации BRAF [28]. Ниволумаб назначался в дозе 3 мг/кг каждые 2 недели до прогрессирования или непереносимой токсичности. В группе сравнения пациенты получали стандартную терапию дакарбазином 1000 мг/м² 
Таблица 1. Эффективность ниволумаба в первой линии терапии неоперабельной или метастатической меланомы без мутации BRAF в исследовании CheckMate 066 [28]

\begin{tabular}{|c|c|c|c|}
\hline Показатели & $\begin{array}{c}\text { Ниволумаб } \\
n=210\end{array}$ & $\begin{array}{c}X T \\
n=208\end{array}$ & Статистическая значимость \\
\hline Медиана ОВ, мес. & НД & $10,8(9,3-12,1)$ & OP $0,42(0,25-0,73) *$ \\
\hline 1 -летняя ОВ, \% & $72,9(65,5-78,9)$ & $42,1(3,0-50,9)$ & - \\
\hline Медиана ВБП, мес. & $5,1(3,5-10,8)$ & $2,2(2,1-2,4)$ & OP $0,43(0,34-0,56) *$ \\
\hline Ч०О, \% & $40,0(33,3-47,0)$ & $13,9(9,5-19,4)$ & ОШ 4,06 $(2,52-6,54)^{*}$ \\
\hline Медиана длительности ответа, мес. & НД & 6,0 (3,3 - НД) & - \\
\hline Медиана времени до ответа, мес. & $2,1(1,6-7,6)$ & $2,1(1,8-3,6)$ & - \\
\hline
\end{tabular}

OP - отношение рисков, ОШ - отношение шансов, НД - не достигнуто

* $p<0,001$ в пользу ниволумаба в сравнении с дакарбазином

каждые 3 недели. Группы были сопоставимы по исходным характеристикам: 61\% пациентов имели стадию M1c, $37 \%$ - ЛДГ выше ВГН, у 35\% уровень экспрессии PD-L1 был $5 \%$ и выше. В качестве основного показателя эффективности была выбрана оценка ОВ по Каплану-Майеру. Оценка опухолевого ответа проводилась по критериям RECIST 1.1.

На момент первичного анализа медиана ОВ была значительно лучше в группе ниволумаба в сравнении с дакарбазином, причем ниволумаб снизил относительный риск смерти на $58 \%$ (см. табл. 1). Медиана ОВ также была выше в группе ниволумаба при подгрупповом анализе, независимо от уровня PD-L1, стадии M, исходного уровня ЛДГ и наличия метастазов в головном мозге. В данных подгруппах снижение риска смерти составило 52-70\% на основании оценки относительного риска [28].

При оценке дополнительных показателей (ВБП, ЧОО) результаты были также в пользу ниволумаба (табл. 1). ЧОО в группе ниволумаба практически в три раза превышала этот показатель группы дакарбазина. Риск прогрессирования заболевания на терапии ниволумабом был на $57 \%$ ниже в сравнении с терапией дакарбазином (табл. 1). Ниволумаб улучшает ЧОО, независимо от уровня экспрессии PD-L1 на опухолевых клетках (при уровне экспрессии PD-L1 $\geq 5 \%$ ЧОО составила $52,7 \%$ и 10,8\%, при уровне экспрессии PD-L1 <5\% - 33,1\% и 15,7\% в группах с ниволумабом и дакарбазином соответственно) [28].

Таким образом, у пациентов с отсутствием в опухоли BRAF мутации ниволумаб значимо увеличивает как частоту объективных ответов и выживаемость без прогрессирования, так и общую выживаемость по сравнению со стандартной химиотерапией независимо от уровня экспрессии PD-L1.

\section{Комбинированная терапия}

Понимание механизмов активации противоопухолевого иммунитета и роли ингибиторов блокаторов иммунного ответа позволило с успехом применять их в клинической практике. В то же время разные группы ингибиторов действуют на разных этапах активации противоопухолевого иммунного ответа. Если ингибиторы CTLA-4 работают на первичном этапе активации, когда опухолевый антиген презентируется неактивным Т-лимфоцитам в лимфатических узлах, то ингибиторы PD-1 работают уже в опухолевом микроокружении в эффекторную фазу: блокируют связывание лиганда PD-L1, экспрессируемого опухолевыми клетками, с PD-1 рецептором цитотоксических Т-лимфоцитов, таким образом, способствуют реализации противоопухолевого иммунитета. Было высказано предположение, что комбинация двух групп препаратов обеспечит более эффективную работу иммунной системы.

В исследовании CheckMate 004, фаза 1, изучалась эффективность и безопасность различных дозовых режимов комбинации ниволумаба и ипилимумаба [20]. При применении ниволумаба в дозе 0,$1 ; 1$ или 3 мг/кг и ипилимумаба в дозах 1 или 3 мг/кг ЧОО достигала 40\% (n=53) у пациентов с меланомой, ранее получавших лечение. Одно- и двухлетняя ОВ составили 85\% и 79\% соответственно. Наиболее высокие показатели одно- и двухгодичной OB достигнуты при комбинации «ниволумаб 1 мг/кг плюс ипилимумаб 3 мг/кг» $(\mathrm{n}=17)$ - 94\% и 88\% соответственно. В расширенной когорте пациентов, получавших ниволумаб 1 мг/кг плюс ипилимумаб 3 мг/кг каждые 3 недели 4 цикла, затем ниволумаб 3 мг/кг каждые 2 недели до прогрессирования $(n=41)$, ЧОО была 44\%, при этом у 7\% пациентов отмечены полные ответы, а у $29 \%$ пациентов таргетные очаги уменьшились более чем на 80\% [20, 29].

На основании полученных данных проведены 2 крупных международных двойных слепых рандомизированных исследования: CheckMate 069, фаза 2 [30, 31], и CheckMate 067, фаза 3 [19, 32], в которых изучалась эффективность и безопасность комбинации ниволумаба и ипилимумаба (ниволумаб 1 мг/кг плюс ипилимумаб 3 мг/кг каждые 3 недели, 4 введения, затем ниволумаб 3 мг/кг каждые 2 недели до прогрессирования или непереносимой токсичности) у пациентов, ранее не получавших лечение с нерезектабельной III или IV стадией меланомы. 
Таблица 2. Эффективность комбинации ниволумаба и ипилимумаба в первой линии терапии неоперабельной или метастатической меланомы в исследованиях CheckMate 069 (фаза 2) [30, 31] и CheckMate $067(\phi а з а ~ 3) ~[19,32]$

\begin{tabular}{|c|c|c|c|c|c|c|c|c|}
\hline \multirow{2}{*}{ Исследование } & \multirow{2}{*}{ Группа } & \multicolumn{3}{|c|}{ Объективный ответ } & \multicolumn{2}{|c|}{ ВБП } & \multicolumn{2}{|c|}{$\mathrm{OB}$} \\
\hline & & Ч००, \% & $\begin{array}{c}\text { ОШ против } \\
\text { Ипи }\end{array}$ & $\begin{array}{l}\text { Медиана } \\
\text { ДО, мес. }\end{array}$ & $\begin{array}{c}\text { Медиана, } \\
\text { мес. }\end{array}$ & $\begin{array}{c}\text { ОР против } \\
\text { Ипи }\end{array}$ & $\begin{array}{l}\text { Медиана, } \\
\text { мес. }\end{array}$ & $\begin{array}{c}\text { OР против } \\
\text { Ипи }\end{array}$ \\
\hline \multirow[t]{3}{*}{ CheckMate 067} & $\begin{array}{c}\text { Ниво }+ \text { Ипи } \\
\text { n=314 }\end{array}$ & $\begin{array}{c}58 \\
(53-64)\end{array}$ & $\begin{array}{c}6,46 \\
(4,45-9,38) \\
p<0,001\end{array}$ & НД & $\begin{array}{c}11,5 \\
(8,7-19,3)\end{array}$ & $\begin{array}{c}0,43 \\
(0,35-0,52) \\
\mathrm{p}<0,001\end{array}$ & НД & $\begin{array}{c}0,55 \\
(0,45-0,69) \\
\mathrm{p}<0,001\end{array}$ \\
\hline & $\begin{array}{c}\text { Ниво } \\
n=315\end{array}$ & $\begin{array}{c}44 \\
(39-50)\end{array}$ & $\begin{array}{c}3,57 \\
(2,48-5,15) \\
\mathrm{p}<0,001\end{array}$ & $\begin{array}{c}\text { НД } \\
(36,3-\text { НД) }\end{array}$ & $\begin{array}{c}6,9 \\
(5,1-9,7)\end{array}$ & $\begin{array}{c}0,55 \\
(0,45-0,66) \\
p<0,001\end{array}$ & $\begin{array}{c}37,6 \\
(29,1-\text { НД })\end{array}$ & $\begin{array}{c}0,65 \\
(0,53-0,80) \\
p<0,001\end{array}$ \\
\hline & $\begin{array}{c}\text { Ипи } \\
n=316\end{array}$ & $\begin{array}{c}19 \\
(15-24)\end{array}$ & - & $\begin{array}{c}19,3 \\
(8,3-\text { НД) }\end{array}$ & $\begin{array}{c}2,9 \\
(2,8-3,2)\end{array}$ & - & $\begin{array}{c}19,9 \\
(16,9-24,6)\end{array}$ & - \\
\hline \multirow[t]{2}{*}{$\begin{array}{l}\text { CheckMate } 069 \\
\text { BRAF ДТ }\end{array}$} & $\begin{array}{c}\text { Ниво +Ипи } \\
\text { n=72 }\end{array}$ & $\begin{array}{c}61 \\
(49-72)\end{array}$ & $\begin{array}{c}12,96 \\
(3,91-54,49) \\
p<0,001\end{array}$ & НД & НД & $\begin{array}{c}0,36 \\
(0,21-0,60) \\
p<0,001\end{array}$ & НД & $\begin{array}{c}0,60 \\
(0,32-1,11)\end{array}$ \\
\hline & $\begin{array}{c}\text { Ипи } \\
\mathrm{n}=37\end{array}$ & $\begin{array}{c}11 \\
(3-25)\end{array}$ & - & НД & $\begin{array}{c}4,4 \\
(2,8-5,7)\end{array}$ & - & $\begin{array}{c}24,8 \\
(10,3-Н Д)\end{array}$ & - \\
\hline \multirow[t]{2}{*}{$\begin{array}{l}\text { CheckMate } 069 \\
\text { BRAF Мyт }\end{array}$} & $\begin{array}{c}\text { Ниво +Ипи } \\
\text { n=23 }\end{array}$ & $\begin{array}{c}52 \\
(31-73)\end{array}$ & - & НД & $\begin{array}{c}8,6 \\
(2,8-\text { НД })\end{array}$ & $\begin{array}{c}0,36 \\
(0,14-0,97) \\
p=0,04\end{array}$ & $\begin{array}{c}\text { НД } \\
(7,1-\text { НД) }\end{array}$ & $\begin{array}{c}1,35 \\
(0,43-4,26)\end{array}$ \\
\hline & $\begin{array}{c}\text { Ипи } \\
\mathrm{n}=10\end{array}$ & $\begin{array}{c}10 \\
(0-45)\end{array}$ & - & НД & $\begin{array}{c}2,7 \\
(1,0-5,4)\end{array}$ & - & $\begin{array}{c}\text { НД } \\
(7,2-\text { НД })\end{array}$ & \\
\hline
\end{tabular}

Ниво - ниволумаб; Ипи - ипилимумаб; чОО - частота объективных ответов; ВБП - выживаемость без прогрессирования; ОВ - общая выживаемость; ДТ - дикий тип; ОР - отношение рисков; ОШ - отношение шансов; НД - не достигнуто

В рандомизированном двойном слепом исследовании CheckMate 069 (фаза 2) изучалась эффективность и безопасность комбинации ниволумаба и ипилимумаба в сравнении с монотерапией ипилимумабом. 24\% пациента имели мутацию BRAF. Основным показателем эффективности стала оценка ЧОО у пациентов с диким типом BRAF мутации. Этот показатель в группе комбинации оказался статистически значимо выше - 61\% (44 из 72) против $11 \%$ (4 из 37) в группе ипилимумаба $(\mathrm{p}<0,001)$ [30]. Полных ответов в группе комбинации было $16 \%$, а в группе ипилимумаба полные ответы не были достигнуты. Медиана длительности ответов не достигнута в обеих группах. Схожие результаты были продемонстрированы и у пациентов с мутацией BRAF (n=33) (табл. 2) [30, 31].

Медиана ВБП при двухлетнем наблюдении в группе комбинации не была достигнута, а в группе ипилимумаба составила 3,0 мес. Одногодичная ВБП составила 52,5\% и $16 \%$, двухлетняя ВБП - 51,3\% и $12 \%$ соответственно в группах комбинации и ипилимумаба. При анализе OB медиана не была достигнута в обеих группах. Одногодичная OB составила $73,4 \%$ в группе комбинации и $63,8 \%$ в группе ипилимумаба (по условиям исследования при прогрессировании на ипилимумабе пациенты могли получать ниволумаб 3 мг/кг каждые 2 недели). Двухгодичная ОВ составила $64,8 \%$ и 53,6\% соответственно [31].

В рандомизированном двойном слепом исследовании 3 фазы CheckMate 067 сравнивалась эффективность применения комбинации ниволумаба и ипилимумаба с монотерапией ниволумабом или ипилимумабом [19]. Уже доступны данные трехлетнего наблюдения [32].
ЧОО в группе комбинации составила 58\% (ПО 19\%), 44\% в группе ниволумаба (ПО 16\%) и 19\% в группе ипилимумаба (ПО 5\%). Медиана длительности ответа не достигнута в обеих группах с ниволумабом, а в группе ипилимумаба составила 19,3 мес. (табл. 2). Медиана ВБП составила 11,5 мес., 6,9 мес. и 2,9 мес. соответственно в группах комбинации, монотерапии ниволумабом и ипилимумабом. Относительный риск прогрессирования при терапии комбинацией снизился на $57 \%$ в сравнении с монотерапией ипилимумабом ( $<<0,001)$, а снижение риска прогрессирования при терапии ниволумабом в сравнении с ипилимумабом ниже на 45\% ( $<0,001)$. Трехлетняя ВБП составила $39 \%$ в группе комбинации, $32 \%$ в группе ниволумаба и $10 \%$ в группе ипилимумаба [32].

На момент первичного анализа при минимальном периоде наблюдения 28 мес. медиана ОВ не была достигнута во всех группах, двухгодичная ОВ составила $64 \%$ в группе комбинации, 59\% в группе ниволумаба и $45 \%$ в группе ипилимумаба. При увеличении срока наблюдения (минимум 36 мес.) медиана ОВ не была достигнута в группе, получавшей комбинацию ниволумаба и ипилимумаба, и составила 37,6 мес. в группе монотерапии ниволумабом и 19,9 мес. в группе ипилимумаба. Показатели трехлетней ОВ составили 58\% в группе комбинации, $52 \%$ в группе ниволумаба и $34 \%$ в группе ипилимумаба. Относительный риск смерти на комбинированной терапии в сравнении с ипилимумабом ниже на $45 \%(\mathrm{p}<0,001)$, риск смерти при терапии ниволумабом в сравнении с ипилимумабом ниже на $35 \%(\mathrm{p}<0,001)$. При дескриптивном анализе отношение риска прогрессирования 
или смерти при сравнении групп комбинации и монотерапии ниволумабом составило 0,78. Последующую системную терапию при прогрессировании получали $32 \%$ пациентов в группе комбинации, 46\% в группе ниволумаба и $63 \%$ в группе ипилимумаба [32].

$\mathrm{y}$ пациентов с наличием мутации BRAF медиана $\mathrm{OB}$ не достигнута в группе комбинации и ниволумаба, а в группе ипилимумаба составила 24,6 мес. Трехлетняя OB у этих пациентов составила $68 \%$ в группе комбинации и $56 \%$ в группе ниволумаба. Для пациентов с диким типом BRAF медиана OB достигнута во всех группах $(39,1 ; 35,8$ и 18,5 мес. соответственно в группах комбинации, ниволумаба и ипилимумаба) [32].

Проводился дополнительный анализ влияния на эффективность терапии уровня экспрессии PD-L1 на опухолевых клетках в группах комбинации ниволумаба и ипилимумаба и монотерапии ниволумабом. При экспрессии PD-L1 <1\% лучшие результаты по общей выживаемости достигаются в группе комбинации: медиана ОВ не достигнута при трехлетнем наблюдении, а в группе ниволумаба она составила 23,5 мес. При экспрессии PD-L1 $\geq 1 \%$ медиана ОВ не достигнута в обеих группах [32].

\section{Безопасность}

Безопасность монотерапии ниволумабом проанализирована в совокупном анализе 4 клинических исследований, включающих 2 исследования 3 фазы [33]. Все пациенты получали ниволумаб в дозе 3 мг/кг каждые 2 недели. На момент анализа 149 пациентов умерли, основной причиной смерти стало прогрессирование заболевания (140 из 149). Среди других причин смерти - острый инфаркт миокарда, сердечно-легочная недостаточность, сердечная недостаточность, гипоксия, сепсис с полиорганной недостаточностью, субарахноидальное кровоизлияние и тромбоэмболия легких. О смертях, связанных с токсичностью ниволумаба, не сообщается.

Из 576 включенных в анализ пациентов у 71\% наблюдались нежелательные реакции (НР), связанные с терапией. Наиболее частыми были следующие: слабость (25\%), зуд (17\%), диарея (13\%) и сыпь (13\%). У 10\% пациентов (57 из 576) наблюдались НР 3-4 степени.

Нежелательные реакции, которые, по мнению исследователей, носили иммуно-опосредованный характер, наблюдались у 49\% пациентов. Наиболее частыми были НР со стороны кожи, желудочно-кишечного тракта, эндокринной системы и печени. Из них НР 3-4 степени наблюдались менее чем в $4 \%$ случаев.

НР, приведшие к прекращению терапии, были выявлены у 17 пациентов (3\%), среди них наиболее частыми оказались колит, повышение АЛТ, повышение липазы и пневмонит (у 2 пациентов). Медиана времени появления НР варьировала от 5 недель (со стороны кожи) до 15 не- дель (со стороны почек). Системные кортикостероиды для купирования НР применялись у 24\% пациентов (114 из 474 пациентов в 2-х исследованиях 3 фазы), в 16\% случаев - топические стероиды, в том числе в $1 \%$ случаев - ингаляционные формы. Назначение более сильных иммуносупрессивных препаратов потребовалось в 0,6\% случаев (3 пациента): инфликсимаб для терапии артрита 3 степени и повышения липазы 4 степени в исследовании CheckMate 037 и микофенолата мофетил при гепатите 3 степени в исследовании CheckMate 066 у пациента, получавшего после терапии ниволумабом ипилимумаб в качестве последующей линии терапии [33].

Интересно отметить, что, как и в исследованиях с ипилимумабом, у пациентов с НР любой степени тяжести ЧОО была выше в сравнении с пациентами, у которых лечение не сопровождалось развитием НР. При этом ЧОО не отличалась у пациентов, которые получали кортикостероиды для купирования НP, и у пациентов, не получавших кортикостероиды [33].

В исследовании CheckMate 067 сравнивалась безопасность применения комбинации ниволумаба и ипилимумаба с монотерапией ниволумабом и ипилимумабом [32]. В группе комбинации частота НР была выше, чем в группах монотерапии. Частота всех НР, связанных с лечением, в исследовании CheckMate 067 была 96\% в группе комбинации, $86 \%$ в группе ниволумаба и $86 \%$ в группе ипилимумаба. Нежелательные реакции 3-4 степени наблюдались у $59 \%$ пациентов в группе комбинации, в $21 \%$ и $28 \%$ случаев соответственно при монотерапии ниволумабом и ипилимумабом. Нежелательные реакции, приведшие к прекращению терапии в группе комбинации, наблюдались чаще: НP 3-4 степени, приведшие к прекращению терапии, регистрировались у $30 \%$ пациентов в группе комбинации, у 8\% в группе ниволумаба и у 14\% пациентов в группе ипилимумаба. Всего было зарегистрировано 2 случая смерти в связи с НР: одна - в группе ниволумаба (нейтропения) и одна - в группе ипилимумаба (перфорация кишки). В группе комбинации ниволумаба и ипилимумаба зарегистрировано 2 смерти, связанных, по мнению исследователей, с лечением, однако обе смерти наступили спустя более чем 100 дней после введения последней дозы ниволумаба (одна - от сердечной недостаточности, обусловленной миокардитом, спустя 433 дня после окончания терапии, вторая - от некроза печени спустя 234 дня после окончания терапии).

В целом на фоне комбинации ниволумаба и ипилимумаба НР возникают чаще в сравнении с монотерапией ниволумабом или ипилимумабом, однако не зарегистрировано новых НР, которые не были бы описаны в других исследованиях, а при следовании специально разработанным алгоритмам большинство НР управляемы и легко купируются, лишь небольшая доля пациентов требует назначения, помимо стероидов, более сильных иммуносупрессантов [32, 33]. 


\section{Заключение}

За последнее десятилетие произошел значительный прогресс в терапии метастатической меланомы. Ипилимумаб впервые продемонстрировал улучшение общей выживаемости ранее леченных пациентов с метастатической меланомой [17]. Таргетные препараты, ингибиторы BRAF и MEK позволяют добиваться объективных ответов у 50-70\% пациентов. В то же время в связи с развитием резистентности к терапии большинство пациентов прогрессируют в среднем через 7-12 месяцев [9, 12].

Ингибиторы PD-1 (ниволумаб и пембролизумаб), по сравнению с монотерапией ипилимумабом, продемонстрировали еще более высокую эффективность. По частоте ответов приближаясь к эффективности таргетных препаратов, они обладают неоспоримым преимуществом - возможностью длительного удержания достигнутых ответов, а также благоприятным профилем безопасности. Комбинация ниволумаба и ипилимумаба улучшила частоту объективных ответов, безрецидивную и общую выживаемость в сравнении с монотерапией ниволумабом и ипилимумабом. В отдельных подгруппах пациентов (экспрессия PD-L1 < $1 \%$, наличие BRAF мутации) комбинация демонстрирует значительное преимущество над монотерапией ниволумабом и ипилимумабом. Переносимость комбинации несколько хуже в сравнении с монотерапией, однако все НР легко управляемы и обратимы при следовании разработанным алгоритмам купирования НР. В то же время данные свидетельствуют, что прекращение терапии в связи с НР не ухудшает эффективность лечения, более того, пациенты с НР имеют более высокие показатели эффективности в сравнении с пациентами, не имеющими НР.

Таким образом, ингибиторы PD-1, и в частности ниволумаб, как в режиме монотерапии, так и в комбинации с ипилимумабом, становятся новым стандартом терапии метастатической меланомы в первой линии, независимо от статуса мутации BRAF или уровня экспрессии PD-L1.

\title{
Информация об авторах
}

Галина Ю. Харкевич, к. М. н., в.н.с. отделения биотерапии опухолей, e-mail: gkharkevich@mail.ru

Лев В. Демидов, д. м. н., профессор, руководитель отделения биотерапии опухолей, e-mail: demidov.lev@gmail.com

DOI:10.18027 / 2224-5057-2017-7-3-62-69

For citation: Kharkevich G. Y., Demidov. L. V. Efficacy of nivolumab in advanced melanoma. Malignant Tumours 2017; 3: 62-69. (In Russ.)

\section{Efficacy of nivolumab in advanced melanoma}

\author{
G. Y. Kharkevich, L. V. Demidov \\ N. N. Blokhin Russian Cancer Research Center, Moscow, Russia
}

\begin{abstract}
Check-point inhibitors are new agents that has significantly improved clinical outcomes in treatment of advanced melanoma treatment. Ipilimumab was the first agent that increased overall survival of pretreated patients with advanced melanoma. Nivolumab is PD-1 inhibitor, studied in three trials of $3^{\text {rd }}$ phase at the dose $3 \mathrm{mg} / \mathrm{kg}$ IV every 2 weeks. Nivolumab monotherapy has median $0 \mathrm{~S}$ equal to 37,6 months, Median OS of nivolumab plus ipilimumab has not been reached in minimum follow up of 36 months. 3 years $0 \mathrm{~S}$ is $58 \%$ in combination group and $52 \%$ in nivolumab group compared to $34 \%$ in ipilimumab group. ORR was $45 \%$ in nivolumab group and $58 \%$ in combination group. In patients with low expression of PD-L1 and BRAF MT combination of nivolumab and ipilimumab has better outcomes. Nivolumab is well tolerated, grade 3-4 adverse reactions amount to $10 \%$ in pooled analysis. Grade 3-4 adverse reactions showed $55 \%$ in combination of nivolumab plus ipilimumab; however, no new types of reactions reported. Majority of adverse reactions were resolved in 3-4 weeks by using special guidelines of adverse reactions management. PD-1 inhibitors, including nivolumab in monotherapy and in combination with ipilimumab become new standard of care for advance melanoma regardless of BRAF status and PD-L1.
\end{abstract}

Keywords: advanced melanoma, check-point inhibitor, immunotherapy, PD-1, CTLA-4 


\section{Information about the authors}

Galina Y. Kharkevich, MD, PhD, Department of Tumor Biotherapy, e-mail: gkharkevich@mail.ru

Lev V. Demidov, MD, PhD, Prof., Head of the Department of Tumor Biotherapy, e-mail: demidov.lev@gmail.com

\section{Литература • References}

1. Thompson J. F., Scolyer R. A., Kefford R. F. Cutaneous melanoma, Lancet, 2005, Vol. 365, No. 9460, pp. 687-701.

2. de Vries E., Bray F. I., Coebergh J. W., Parkin D. M. Changing epidemiology of malignant cutaneous melanoma in Europe 1953-1997: rising trends in incidence and mortality but recent stabilizations in Western Europe and decreases in Scandinavia, Int. J. Cancer, 2003, Vol. 107, No. 1, pp. 119-126.

3. World Health Organization. Skin cancers. Available from: http://www.who.int/uv/faq/skincancer/en/index1. html, Accessed March 11, 2017.

4. Balch C. M., Soong S. J., Gershenwald J.E. et al. Prognostic factors analysis of 17,600 melanoma patients: validation of the American Joint Committee on Cancer melanoma staging system, J. Clin. Oncol., 2001, Vol. 16, No. 19, pp. 3622-3634.

5. Barth A., Wanek L. A., Morton D. L. Prognostic factors in 1,521 melanoma patients with distant metastases, J. Am. Coll. Surg., 1995, Vol. 181, No. 3, pp. 193-103.

6. Каприн А. Д., Старинский В. В., Петрова В. В. Состояние онкологической помощи населению России в 2016 году. Москва: МНИОИ им. П. А. Герцена - филиал ФГБУ «НМИРЦ» Минздрава России, 2017. 266 с. [Kaprin A. D., Starinskiy V. V., Petrova V. V. Sostoyanie onkologicheskoy pomoshchi naseleniyu Rossii v 2016 godu, Moscow: MNIOI im. P. A. Gertsena - filial FGBU "NMIRTs" Minzdrava Rossii, 2017, 266 p.]

7. Fletcher W.S., Pommier R. F., Lum S., Wilmarth T. J. Surgical treatment of metastatic melanoma, Am. J. Surg, 1998, Vol. 175, No. 5, pp. 413-417.

8. Lui P., Cashin R., Machado M., Hemels M. et al. Treatments for metastatic melanoma: synthesis of evidence from randomized trials, Cancer Treat Rev., 2007, Vol. 33, No. 8, pp. 665-680.

9. Maverakis E., Cornelius L. A., Bowen G. M. et al. Metastatic melanoma: a review of current and future treatment options, Acta Derm. Venereol., 2015, Vol. 95, No. 5, pp. 516-524.

10. Hall R. D., Kudchadkar R. R. BRAF mutations: signalling, epidemiology, and clinical experience in multiple malignancies, Cancer Control, 2014, Vol. 21, No. 3, pp. 221-230.

11. Giovanni P., Giovanni P., Aldo T. et al. Molecular targeted approaches for advanced BRAF V600, N-RAS, C-KIT and GNAQ melanomas, Dis Markers, 2014, No. 2014, 671283.

12. Sullivan R., LoRosso P., Boerner S., et al. Achievements and challenges of molecular targeted therapy in melanoma, Am. Soc. Clin. Oncol. Educ. Book, 2015, No. 35, pp. 177-186.

13. Luke J. J., Ott P. PD-1 pathway inhibitors: the next generation of immunotherapy for advanced melanoma, Oncotarget, 2015, Vol. 6, No. 6, pp. 3479-3492.

14. Antonia S. J., Larkin J., Ascierto P. A. Immuno-oncology combinations: a review of clinical experience and future prospects, Clin. Cancer Res., 2014, Vol. 20, No. 24, pp. 6258-6568.

15. Shih K., Arkenau H. T., Infante J. R. Clinical impact of checkpoint inhibitors as novel cancer therapies, Drugs, 2014, Vol. 74, No. 17, pp. 1993-2013.

16. Tsai K. K., Daud A. L. The role of anti-PD-1/PD-L1 agents in melanoma: progress to date, Drugs, 2015, Vol. 75, No. 6, pp. 563-575.

17. Hodi F. S., O'Day S. J., McDermott D. F. et al. Improved survival with ipiipilimumab in patients with metastatic melanoma, N. Engl. J. Med., 2010, Vol. 363, No. 8, pp. 711-723.

18. Deeks E. D. Nivolumab: a review of its use in patients with malignant melanoma, Drugs, 2014, Vol. 74, No. 11, pp. $1233-1239$.

19. Larkin J., Chiarion-Sileni V., Gonzalez R. et al. Combined nivolumab and ipilumumab or monotherapy in untreated melanoma, N. Engl. J. Med., 2015, Vol. 573, No. 1, pp. 21-34.

20. Wolchok J.D., Kluger H., Callahan M. K. et al. Nivolumab plus ipilimumab in advanced melanoma, N. Engl. J. Med., 2013, Vol. 369, No. 2, pp. 122-133.

21. Ascierto P. A., Melero I., Bhatia S. et al. Initial Efficacy of Anti-Lymphocyte Activation Gene-3 (anti - LAG-3; BMS-986016) in Combination With Nivolumabin Patients With Melanoma Previously Treated With Anti - PD-1/PD-L1 Therapy, J. Clin. Oncol., 2017, No. 35 (suppl.; abstr 9520). 
22. Siu L. L., Steeghs N., Meniawy T. et al. Preliminary Results of a Phase 1/2a Study of BMS-986156 (glucocorticoid-induced tumor necrosis factor receptor - related gene [GITR] agonist) Alone and in Combination With Nivolumab in Patients With Advanced Solid Tumors, 2017, No. 35 (suppl.; abstr 104).

23. Perez R.P., Riese J. M., Lewis K. D. et al. Epacadostat plus nivolumab in patients with advanced solid tumors: Preliminary phase I/II results of ECHO-204, J. Clin. Oncol., 2017, No. 35, (suppl; abstr 3003).

24. Topalian S. L., Hodi S. F., Brahmer J. R. et al.. Safety, Activity, and Immune Correlates of Anti - PD-1 Antibody in Cancer, N. Engl. J. Med., 2012, Vol. 366, No. 26, pp. 2443-2453.

25. Hodi F. S., Kluger H., Sznol M. et al. Durable, long-term survival in previously treated patients with advanced melanoma (MEL) who received nivolumab (NIVO) monotherapy in a phase I trial, Oral presentation at: AACR Annual Meeting 2016, abstr. CT001.

26. Weber J.S., D'Angelo S.P., Minor D. et al. Nivolumab versus chemotherapy in patients with advanced melanoma who progressed after anti-CTLA-4 treatment (CheckMate 037): a randomised, controlled, open-label, phase 3 trial, Lancet Oncol., 2015, Vol. 16, No. 4, pp. 375-384.

27. Larkin J., Minor D., D’Angelo S. et al. Overall Survival in Patients With Advanced Melanoma Who Received Nivolumab Versus Investigator's Choice Chemotherapy in CheckMate 037: A Randomized, Controlled, Open-Label Phase III Trial, J. Clin. Oncol., 2017 Jul 3, JC02016718023 [Epub ahead of print].

28. Robert C., Long G. V., Brady B. et al. Nivolumab in previously untreated melanoma whithout BRAF mutation, N. Eng. J. Med., 2015, Vol. 372, No. 4, pp. 320-330.

29. Kluger H. M., Sznol M., Callahan M. K. et al. Survival, response duration, and activity by BRAF mutation status in a phase 1 trial of nivolumab (anti-PD-1; BMS-936558, 0N0-4538) and ipilimumab concurrent therapy in advanced melanoma (MEL) [abstract], Pigment Cell Melanoma Res., 2014, Vol. 27, No. 6, p. 1203.

30. Postow M. A., Chesney J., Pavlick A. C. et al. Nivolumab and ipilimumab versus ipilimumab in untreated melanoma, N. Engl. J. Med., Vol. 372, No. 21, pp. 2006-2017.

31. Hodi F. S., Chesney J., Pavlick A. C. et al. Combined nivolumab and ipilimumab versus ipilimumab alone in patients with advanced melanoma: 2-year overall survival outcomes in a multicentre, randomised, controlled, phase 2 trial, lancet Oncol., 2016, Vol. 17, No. 11, pp. 1558-1568.

32. Wolchok J. D., Chiarion-Sileni V., Gonzales R. et al. Overall Survival with Combined Nivolumab and Ipilimumab in Advanced Melanoma, N. Engl. J. Med., Sep 2017, pp. 12. DOI: 10.1056/NEJMoa1709684. [Epub ahead of print].

33. Weber J. S., Hodi F. S., Wolchjok J. D. et al. Safety Profile of Nivolumab Monotherapy: A Pooled Analysis of Patients With Advanced Melanoma, J. Clin. Oncol., 2017, Vol. 35, No. 7, pp. 785-791. 